\title{
Methodology and timing of standardization
}

\author{
Luca Bertolaccini ${ }^{1}$, Gaetano Rocco ${ }^{2}$ \\ ${ }^{1}$ Department of Thoracic Surgery, Maggiore Teaching Hospital, Bologna, Italy; ${ }^{2}$ Department of Surgery, Memorial Sloan Kettering Cancer Center, \\ New York, NY, USA \\ Correspondence to: Luca Bertolaccini, MD, PhD, FCCP. Department of Thoracic Surgery, Maggiore Teaching Hospital, Largo Nigrisoli 2, 40133 \\ Bologna, Italy. Email: luca.bertolaccini@gmail.com.
}

\begin{abstract}
In the last decade, uniportal VATS has increasingly been used for major lung resections as it has revolutionised the way thoracic surgeons treat pulmonary lesions. Uniportal VATS has represented an authentic innovation in thoracic surgery because it represents an ideal link between reduced morbidity and enhanced recovery. From the first feasibility studies, Uniportal VATS has been assessed in terms of postoperative morbidity, mortality, and overall oncologic value yielding similar outcomes compared to open thoracotomy or three-port VATS. The Uniportal VATS Interest Group (UVIG) is a working group inside the European Society of Thoracic Surgeons (ESTS) with a particular interest in Uniportal VATS as the preferred approach to intrathoracic conditions amenable to surgical diagnosis and treatment. The first aim of UVIG was to define a consensus statement on the indications and techniques of uniportal VATS. The first consensus paper among international experts in uniportal VATS lobectomy was obtained through a Delphi process through three rounds of voting to enhance its validity. The Delphi process was used to define the procedure and to optimise the indications, perioperative management and training of Uniportal VATS. The main features of this process including the anonymity of participants, the iterative process, and, controlled feedback were reported in the consensus statement.
\end{abstract}

Keywords: Uniportal VATS; lung cancer; methodology; Delphi technique

Submitted Nov 18, 2018. Accepted for publication Feb 14, 2019.

doi: $10.21037 /$ jtd.2019.02.35

View this article at: http://dx.doi.org/10.21037/jtd.2019.02.35

\section{Introduction}

Uniportal video-assisted thoracoscopic surgery (UniVATS) seems a feasible approach to lung cancer and other intrathoracic conditions. However, the eye-hand coordination and the approach to the target lesion could represent challenging steps in the learning curve. Consensus on the indications and surgical technique is lacking even though the majority of the surgeons regularly performing UniVATS lobectomy seem to have elected an anterior approach (1). The Uniportal VATS Interest Group (UVIG) is a working group inside the European Society of Thoracic Surgeons (ESTS) with a particular interest in UniVATS as the preferred approach to intrathoracic conditions amenable to surgical diagnosis and treatment. UVIG's first goal was to establish a consensus based on the more than 200 papers on UniVATS published in PubMed which did not include any guidelines or recommendations. Therefore, despite a significant number of articles, there are still many doubts and controversies about the definition of the procedure, the optimisation of indication and the postoperative management of UniVATS. In this paper, we aim to describe the methodology of the consensus statement about UniVATS.

\section{Consensus methods}

Trying to define a consensus when there is an overload of information is a well-known situation in medical research. Meta-analyses (quantitative methods) have been developed to achieve statistical summaries of the results of trials and to resolve inconsistencies in study findings. Consensus methods enable the visions of experts to the decisions. Two consensus techniques were generally accepted in medical research: the nominal group technique (expert panel) 
Table 1 Methodologic criteria reported in the consensus statements achieved with the Delphi technique (4)

\begin{tabular}{ll}
\hline Study objective & List of a statement reflecting the consensus of the group or the level of agreement \\
\hline Participants & Selections of participants \\
Consensus definition & Definition of the threshold value required for the Delphi \\
Delphi process & Definition of the specific number of Delphi rounds \\
& Definition of criteria used to determine which items to drop \\
\hline
\end{tabular}

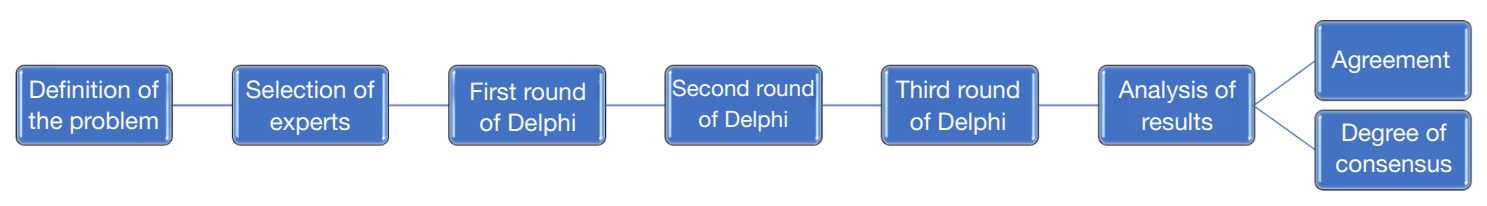

Figure 1 The process of a consensus using the Delphi technique. To scoring the agreement with statements, respondents are asked to rate the confidence or express their opinions.

and the Delphi process. These methods attempt to assess the consensus measurement (amount of agreement) and the consensus development (resolution of disagreement). Consensus approaches aim to define about an assumed subject the range to which experts agree. They overwhelmed some of the disadvantages usually found with decision making in committees (frequently subjugated by one or more individuals) or in open committees where subjects are frequently not ready to publicly state their opinions. Therefore, the term agreement could have two formulae. The amount to which each subject agrees with the question under study (valued on a categorical or numerical scale); the level to which experts reach an agreement with each other (assessed by measures of average and dispersion) (2).

Various factors influenced the choice between the nominal group technique or the Delphi technique (e.g., the insight of consensus required, the research question, the related feasibilities, time limitations, and geography). If investigators are looking to explore ideas about a topic or an enquiry, this best bring into line with the nominal group technique. In case of development of guidelines, a Delphi technique including experts of the field would be more appropriate. The progress of guidelines, requiring a more demanding procedure with consensus from a more significant number of experts, is more comfortable with the Delphi technique. Also, the nominal group technique necessitates frontal meetings and could be more culturally suitable, even if experts are at a distance. Nevertheless, it may be more challenging to arrange a formal meeting of the experts for a preferred time (3).
Therefore, regarding the consensus about UniVATS characteristics, we decide to use the Delphi methodology to simplifies the development and measurement of consensus among experts. The main features of this process including the anonymity of participants, the iterative process, and, controlled feedback in the consensus statements (Table 1).

\section{The Delphi technique}

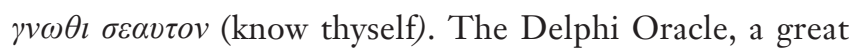
historical importance woman, still shrouded in mystery, inspires the name of Delphi technique. The Oracle spoke for the god Apollo and responded enquiries for the Greeks and foreign inquirers.

In methodology, the Delphi technique is a broadly accepted method used for accomplishing conjunction of opinion concerning knowledge asked from experts within specific topic areas and was developed by Dalkey and Helmer at the Rand Corporation in the first half of last century. Created on the basis that two or many heads are better than one, the Delphi technique is a communication procedure aimed to conduct general thoughts of a precise issue for goal scenery, policy examination, or forecasting the incidence of future events. Delphi technique can be used to control or progress a range of thinkable replacements; to discover fundamental assumptions or information due to diverse findings; to pursue information generating a consensus on part of the experts; to compare knowledgeable decisions on a topic across a widespread range of disciplines; and to teach the group to different aspects of the topic (5). 
The Delphi technique uses an extremely organised meeting to gather information from experts of a given issue (Figure 1). In round 1, either the experts are requested to offer opinions on a specific topic, based on their knowledge and experience. These thoughts are assembled under a limited number of statements drafted to all experts on a questionnaire. In round 2, experts rank their agreement with each statement in the questionnaire. The rankings are summarised and included in a repeat version of the questionnaire. In round 3, experts rank another time their agreement with each statement in the questionnaire, with the opportunity to change their score given the experts response. The new rankings are summarised and evaluated for degree of consensus. If an adequate degree of consensus is gained, the process may conclude with final results feedback (2).

The Delphi process is anonymous for reduction of effects of leading expert and avoiding domination of the consensus process by one or a few experts. Furthermore, the problem of secrecy is facilitated by geographic dispersion of the experts as well as the email usage to solicit and exchange information. Theoretically, the Delphi process can be uninterruptedly reiterated until a consensus is determined to have been accomplished. However, three iterations are in most cases satisfactory to assemble the information and to reach a consensus (5). The Delphi technique is more flexible than the nominal group technique and is accessible to experts unrelatedly of location and avoiding travel expenses if conducted by email.

Nevertheless, the rounds can conclude only after weeks or months (3). Controlled feedback in the Delphi is planned to decrease the noise effect (communication occurred in a group which distorts data), a bias not correlated to the purposes of the study. Through the multiple iterations, subjects should become more problem-solving oriented, offering more insightfully opinions, minimising the effects of noise. Lastly, the ability to use statistical methods additional reduces the possible pressure of the experts for conformity (5).

The Delphi process has been widely used for similar purposes in Literature. For example, the Delphi method was used as a structured process for collecting and distilling knowledge from a group of internationally recognised VATS experts to develop an assessment tool for VATS lobectomy (6). The results of a Delphi process define procedures to optimise robotic training of thoracic surgeons (7).

Cite this article as: Bertolaccini L, Rocco G. Methodology and timing of standardization. J Thorac Dis 2019;11(Suppl 16):S2050-S2052. doi: 10.21037/jtd.2019.02.35

\section{Conclusions}

ESTS-UVIG has organised the first consensus paper among international experts in UniVATS lobectomy was obtained through a Delphi process to define the procedure and to optimise the indications, the perioperative management and training needed for UniVATS. The results of this consensus statement will be published soon.

\section{Acknowledgments}

None.

\section{Footnote}

Conflicts of Interest: The authors have no conflicts of interest to declare.

\section{References}

1. Rocco R, Rocco G. Future study direction on single port (uniportal) VATS. J Thorac Dis 2016;8:S328-32.

2. Jones J, Hunter D. Consensus methods for medical and health services research. BMJ 1995;311:376-80.

3. McMillan SS, King M, Tully MP. How to use the nominal group and Delphi techniques. Int J Clin Pharm 2016;38:655-62.

4. Diamond IR, Grant RC, Feldman BM, et al. Defining consensus: a systematic review recommends methodologic criteria for reporting of Delphi studies. J Clin Epidemiol 2014;67:401-9.

5. Boulkedid R, Abdoul H, Loustau M, et al. Using and reporting the Delphi method for selecting healthcare quality indicators: a systematic review. PLoS One 2011;6:e20476.

6. Jensen K, Petersen RH, Hansen HJ, et al. A novel assessment tool for evaluating competence in videoassisted thoracoscopic surgery lobectomy. Surg Endosc 2018;32:4173-82.

7. Veronesi G, Dorn P, Dunning J, et al. Outcomes from the Delphi process of the Thoracic Robotic Curriculum Development Committee. Eur J Cardiothorac Surg 2018;53:1173-9. 